УДК 536.242

\title{
ВЛИЯНИЕ МАССОВОЙ СКОРОСТИ ПОТОКА НА ХАРАКТЕРИСТИКИ ТЕЧЕНИЯ И ТЕПЛООБМЕНА В ГЛАДКИХ ТРУБАХ ПРИ СВЕРХКРИТИЧЕСКИХ ПАРАМЕТРАХ
}

Фиалко Н.М. ${ }^{1,3}$, член-корреспондент НАН Украины, Пиоро И..Л. ${ }^{2}$, докт. техн. наук, Майсон Н.В. Меранова Н.О.', канд. техн. наук, Шараевский И.Г. ${ }^{3}$, докт. техн. наук

${ }^{1}$ Институт технической теплофизики НАН Украины, ул. Желябова, 2а, Киев, 03057, Украина

${ }^{2}$ Faculty of Energy Systems and Nuclear Science University of Ontario Institute of Technology 2000 Simcoe Str. N., Oshawa ON L1K 7 K4 Canada

${ }^{3}$ Институт проблем безопасности АЭС НАН Украины, ул. Льсогорская, 12, Киев, 03028, Украина

Аналізуються дані комп'ютерного моделювання зі встановлення закономірностей впливу масової швидкості потоку на характеристики течії та теплообміну в гладких трубах при надкритичних параметрах. Представлено результати досліджень, що відповідають зміні масової швидкості від 1002 кг/( $\left.\mathrm{M}^{2} \mathrm{c}\right)$ до 1400 кг/( $\left.{ }^{2} \mathrm{c}\right)$.
Анализируются данные компьютерного моделирования по установлению закономерностей влияния массовой скорости потока на характеристики течения и теплообмена в гладких трубах при сверхкритических параметрах. Представлены результаты исследований, отвечающих изменению массовой скорости от

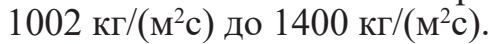

Библ. 11, рис. 6.

Ключевые слова: сверхкритическая вода, течение, теплообмен, массовая скорость, CFD моделирование.

$G$ - массовая скорость, кг/(м² $)$;

$P$ - давление на входе в трубу, МПа;

$q$ - плотность теплового потока на стенке трубы, $\mathrm{BT} / \mathrm{M}^{2}$;

$r$ - радиальная координата, м;

$T$ - температура, ${ }^{\circ} \mathrm{C}$;

$U_{z}$ - осевая компонента скорости, м/с;

$z$ - осевая координата, м;

$\alpha$ - коэффициент теплоотдачи, Вт/( $\left.{ }^{2} \mathrm{~K}\right)$;

$\rho-$ плотность, кг/ $\mathbf{M}^{3}$.

\section{Введение}

В связи с разработкой концепции инновационного реактора АЭС с охлаждающей средой при сверхкритических давлениях актуальными являются исследования, направленные на изучение различных аспектов гидродинамики и теплообмена воды в таких условиях [1-9].

Настоящая работа посвящена CFD прогнозам течения и теплообмена восходящего потока

\section{Нижние индексы:}

$b$ - среднемассовый (bulk fluid);

$d h t$ - ухудшенный теплообмен (deteriorated heat transfer);

$p c$ - псевдокритический (pseudocritical);

$w$-стенка (wall).

\section{Сокращения:}

CFD - Computational Fluid Dynamics;

АЭС - атомная электростанция.

сверхкритической воды в вертикальных гладких трубах при высоких массовых скоростях жидко-

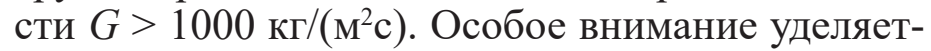
ся изучению влияния величины данной скорости потока на гидродинамические и тепловые характеристики сверхкритической воды.

\section{Постановка задачи и методика исследований}

Особенности постановки задачи смешанной 
теплоотдачи, отвечающей анализируемой физической ситуации, рассмотрены в [10]. Здесь же приводятся численные значения основных величин, входящих в условия однозначности, и характеристики расчетной сетки, применяемой при численной реализации данной задачи с использованием программного обеспечения FLUENT.

В ходе исследований величина массовой скорости изменялась от 1002 кг/( $\left.\mathrm{M}^{2} \mathrm{c}\right)$ до 1400 кг/( $\left.\mathrm{M}^{2} \mathrm{c}\right)$. При этом значения плотности подводимого теплового потока на поверхности стенки трубы и давления воды на входе в трубу оставались постоянными $q=681$ кВт/м², $P=23,9$ МПа.

\section{Результаты исследований и их анализ}

В работе для рассматриваемых вариантов расчетов, отвечающих значениям $G=1002$, 1200 и 1400 кг/( $\left.{ }^{2} \mathrm{c}\right)$, по зависимости [11] были определены величины минимального теплового потока $q_{d h t}$, при превышении которого возникает режим ухудшенного теплообмена. Для указанных значений $G$ эти величины составили 687,835 и $984 \mathrm{\kappa BT} / \mathrm{M}^{2}$, что больше принимаемого в данной работе значения $q$ равного 681 кВт/м². Таким образом, согласно выполненным оценкам, все анализируемые ситуации характеризуются отсутствием режима ухудшенного теплообмена.

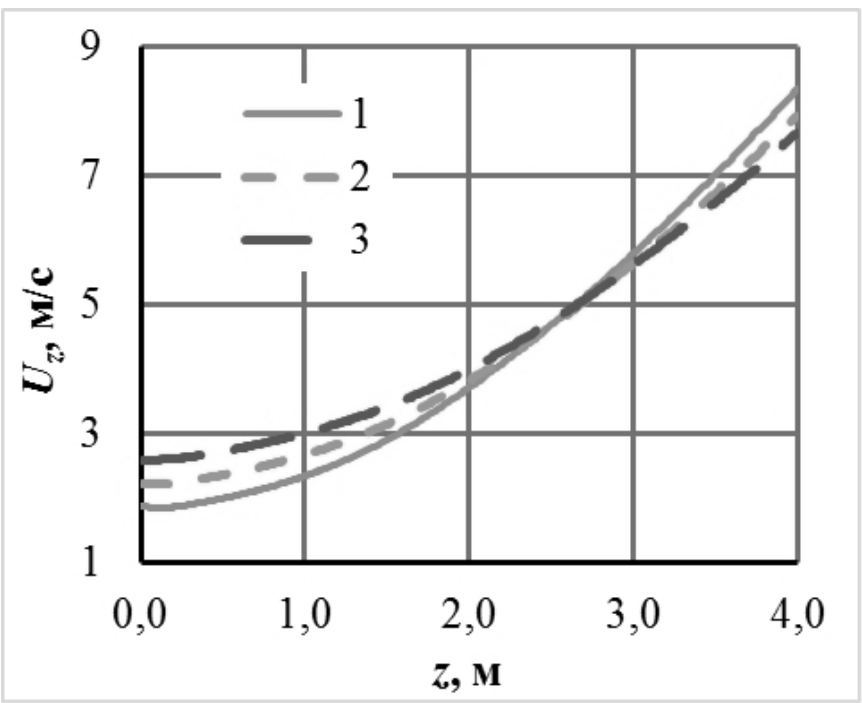

Рис. 1. Распределение осевой компоненты скорости вдоль оси трубы при варьировании величины массовой скорости воды $G$ : $1-G=1002 \kappa z /\left(M^{2} c\right) ; 2-G=1200 \kappa z /\left(M^{2} c\right) ;$ $3-G=1400 \kappa 2 /\left(M^{2} c\right)$.
На рисунках 1-6 приведены характерные результаты выполненных исследований в условиях варьирования массовой скорости сверхкритической воды. При анализе полученных данных рассмотрим вначале особенности влияния этой скорости на соответствующие особенности картины течения и затем для теплообмена.

Рисунки 1 и 2 иллюстрируют результаты компьютерного моделирования, касающиеся закономерностей течения сверхкритической воды при варьировании величины массовой скорости $G$.

Как следует из приведенных данных, влияние $G$ на характеристики течения сверхкритической воды оказываются неоднозначными. Так, на значительном участке трубы, прилежащем к ее входу $(z \approx 2,6$ м), при увеличении $G$ скорость воды на оси трубы повышается. Однако при $z>2,6 \mathrm{M}$ наблюдается обратная картина. Здесь с ростом массовой скорости воды $G$ осевая скорость $U_{z}$, напротив, падает (рис. 1).

Описанный характер влияния массовой скорости связан со следующими двумя конкурирующими факторами. Во-первых, с тем, что увеличение $G$ должно приводить к повышению скорости потока, как это имеет место в изотермических условиях. И, во-вторых, с тем, что при увеличении массовой скорости сверхкритической воды уровень ее нагрева по длине канала становится ниже и соответственно ее плотность вниз по потоку падает менее существенно (рис. 3). Ввиду этого повышение $G$, напротив, должно обусловливать понижение скорости потока. Согласно приведенным данным, при $z \leq 2,6$ м доминирующим является первый из указанных факторов, а при $z>2,6$ м - второй.

Рисунки 4 - 6 иллюстрируют результаты компьютерного моделирования, касающиеся особенностей теплообмена при варьировании массовой скорости $G$. Как видно из рис. $4, a$, изменение величины $G$ весьма существенно влияет на значения коэффициента теплоотдачи $\alpha$. С ростом массовой скорости коэффициент теплоотдачи заметно повышается на всем обогреваемом участке трубы. Так, в центральной части трубы при увеличении $G$ от 1002 кг/(м²) до 1400 кг/( $\left.\mathrm{M}^{2} \mathrm{c}\right)$ значение $\alpha$ повышается в 2,25 раза.

С ростом массовой скорости $G$ несколько изменяется также характер зависимости 
$\alpha=f(z)$. Изменение $\alpha$ по длине трубы становится существенно более неравномерным. При этом локальный максимум $\alpha$ в ее центральной части оказывается более ярко выраженным. Такая

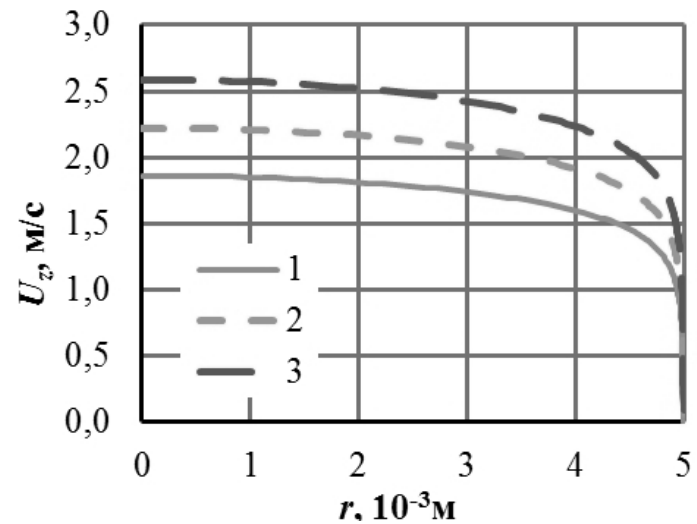

a)

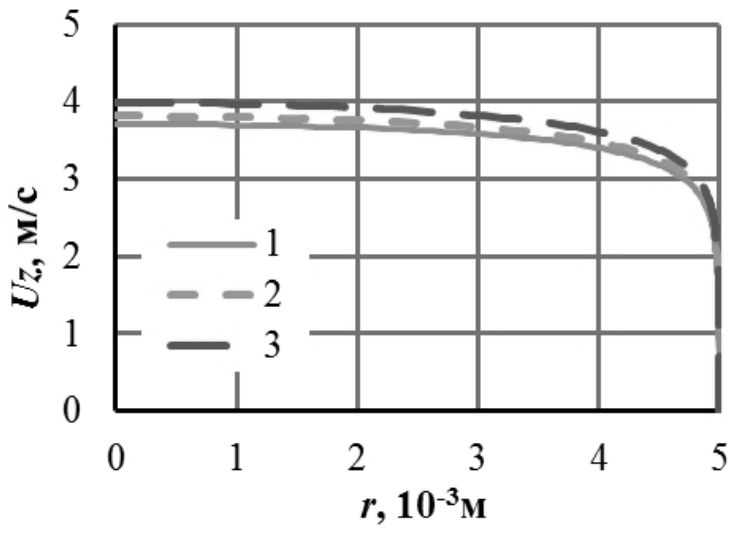

b) картина влияния массовой скорости на величину коэффициента теплоотдачи, как очевидно, определяется при $q=$ const особенностями поведения по длине трубы температур $T_{w}$ и $T_{b}$.

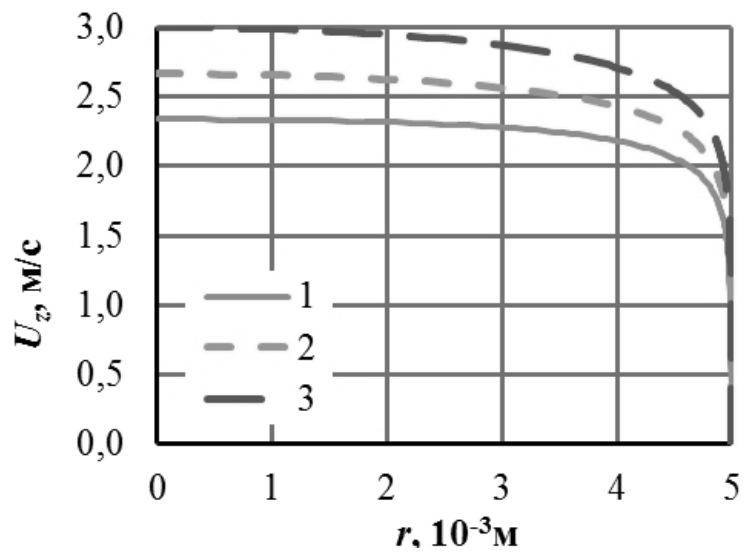

б)

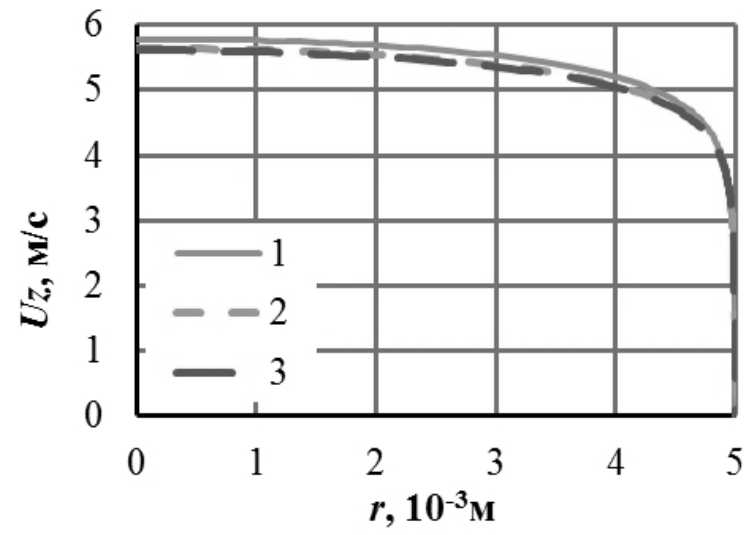

2)

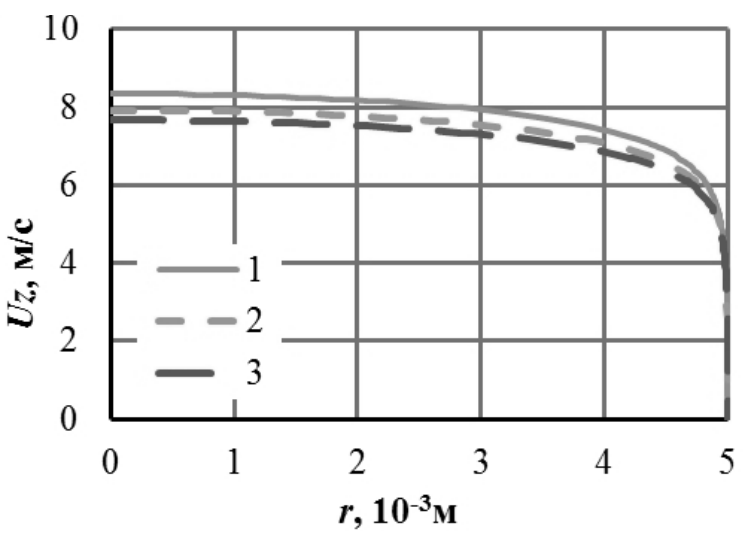

d)

Рис. 2. Профили осевой компоненты скорости в поперечном сечении трубы, отвечающие значениям массовой скорости воды $G=1002$ к2/(м²) - (1);

$G=1200 \kappa z /\left(м^{2} c\right)-(2) ; G=1400 \kappa z /\left(м^{2} c\right)-(3)$, на различном удалении z от входа в ее обогреваемый участок: (а) $-z=0$; (б) $-z=1 \mathrm{~m}$; (в) $-z=2 \mathrm{~m}$; (2) $-z=3 \mathrm{~m}$; (d) $-z=4 \mathrm{M}$. 


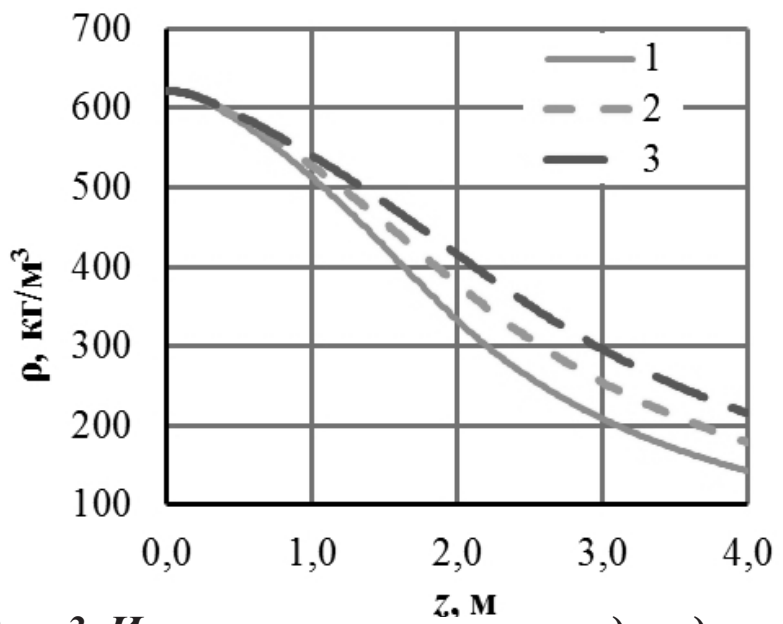

Рис. 3. Изменение плотности воды вдоль оси трубы при различных значениях массовой скорости воды $G: 1-G=1002 \kappa 2 /\left(м^{2} c\right)$; $2-G=1200 \kappa 2 /\left(\boldsymbol{M}^{2} c\right) ; 3-G=1400 \kappa 2 /\left(M^{2} c\right)$.
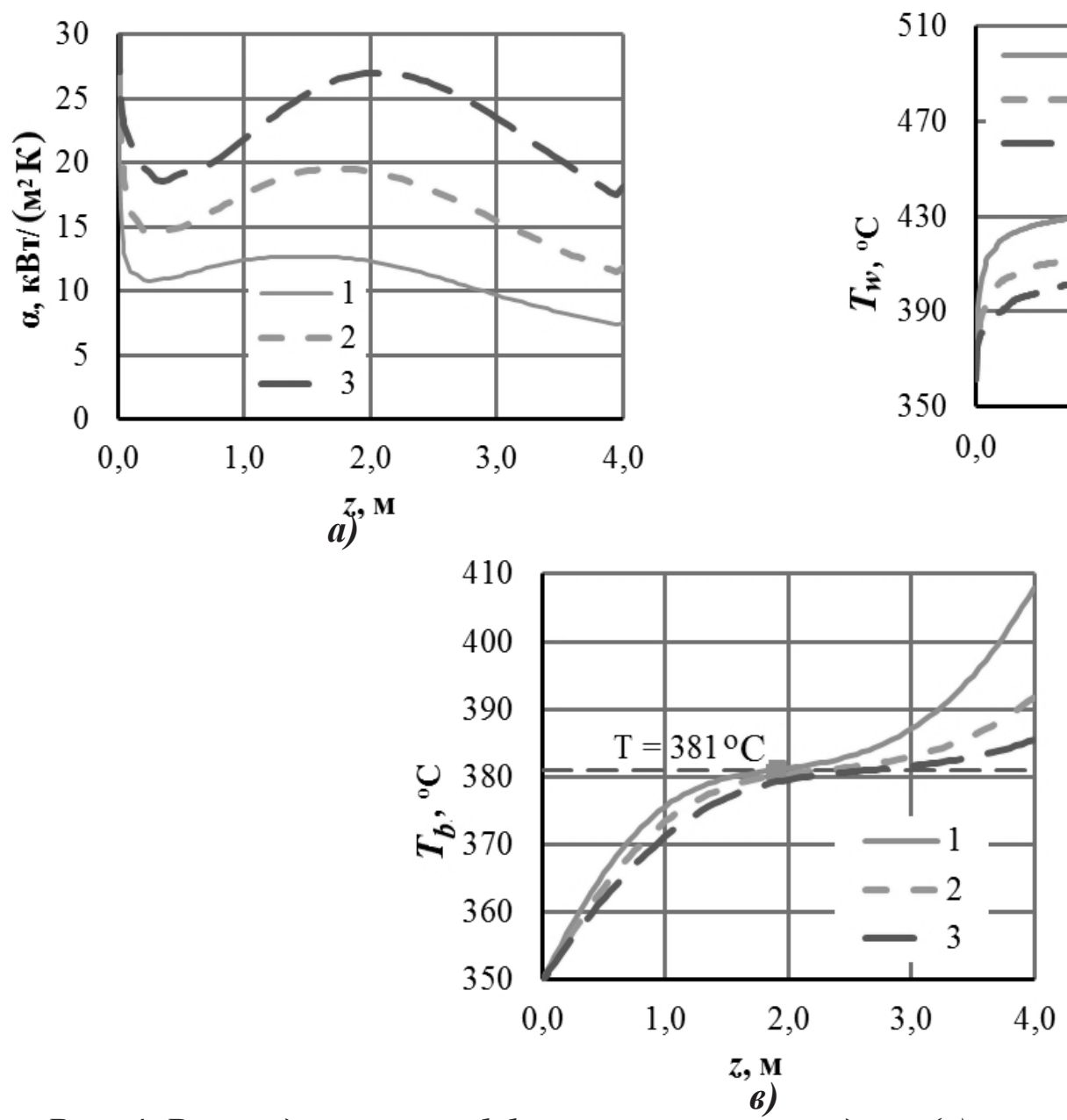

Рис. 4. Распределение коэффициента теплоотдачи (а), температуры $T_{w}$ внутренней поверхности стенки (б) и среднемассовой $T_{b}$ температуры воды (в) вдоль обогреваемого участка трубы при различных значениях массовой скорости воды $G: 1-G=1002 \kappa 2 /\left(\mathrm{M}^{2} c\right) ; 2-G=1200 \kappa 2 /\left(\mathrm{M}^{2} c\right) ; 3-G=1400 \kappa 2 /\left(\mathrm{M}^{2} c\right)$. 


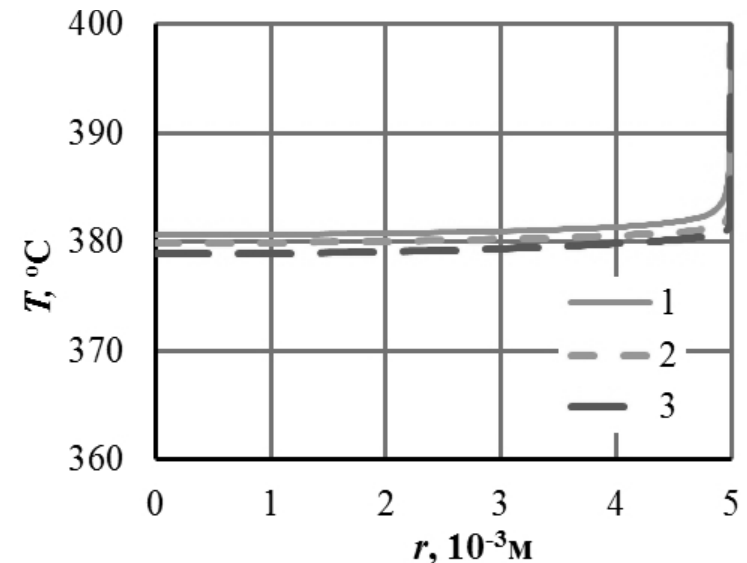

a)

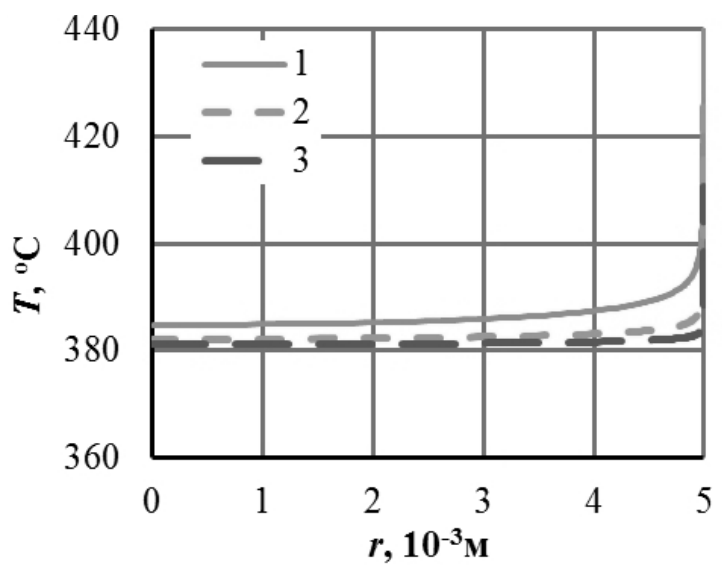

в)

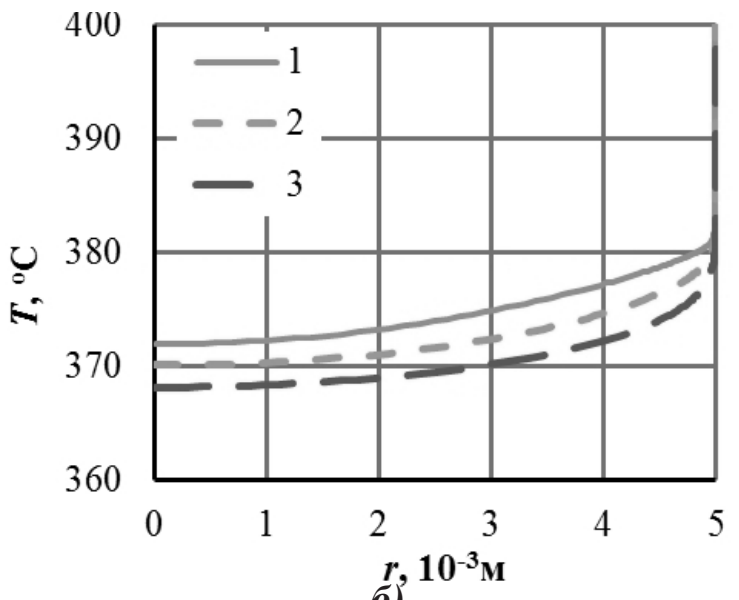

б)

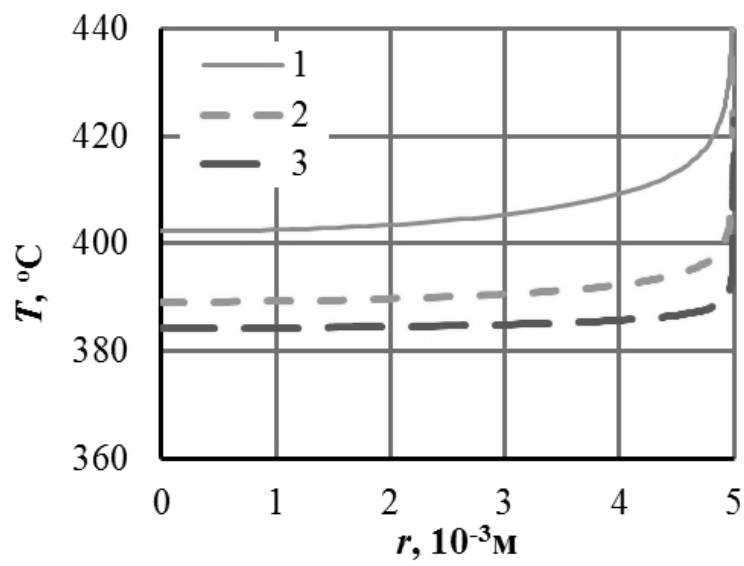

2)

Рис. 5. Профили температуры в поперечном сечении трубы, отвечающие значениям

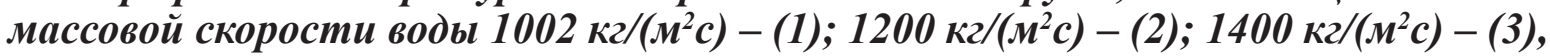
на различном удалении z от входа в ее обогреваемый участок: (a) $-z=1 \mathrm{~m} ;$ (б) $-z=2 \mathrm{~m} ;($ в) $-z=3 \mathrm{~m} ;$ ( () $-z=4 \mathrm{~m}$.

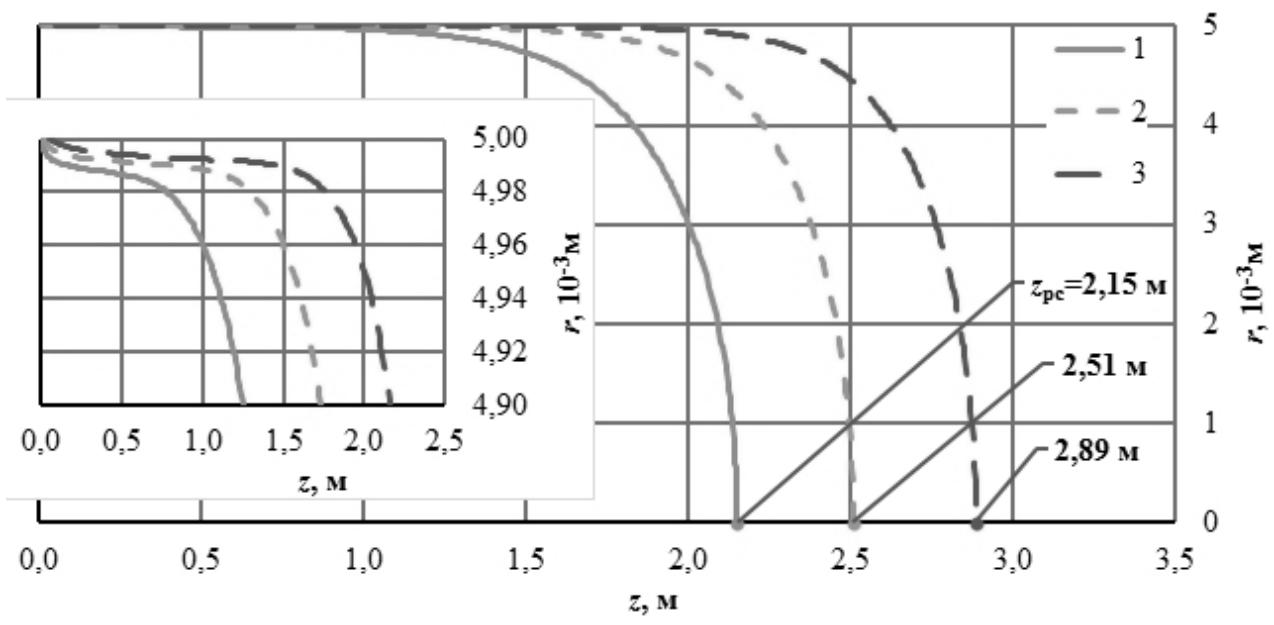

Рис. 6. Конфигурация фронта псевдокритического фазового перехода воды в трубе при различных значениях ее массовой скорости $G$ :

$1-G=1002 \kappa z /\left(M^{2} c\right) ; 2-G=1200 \kappa z /\left(M^{2} c\right) ; 3-G=1400 \kappa z /\left(м^{2} c\right)$. 
То есть в рамках этого участка перепад температур $\Delta T$ падает, что и обусловливает повышение $\alpha$. Дальнейшее по потоку снижение $\alpha$ связано с повышением $\Delta T$, которое определяется достаточно резким ростом температуры стенки при весьма умеренном повышении среднемассовой температуры жидкости.

Картину движения фронта псевдокритического перехода для различных величин массовой скорости иллюстрирует рис. 6. Согласно приведенным данным фронт движется от стенки к оси трубы тем быстрее, чем меньше массовая скорость воды. Он достигает оси трубы при $\mathrm{z}=2,15 \mathrm{м}, 2,51$ м и 2,89 м соответственно для

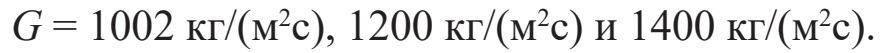

\section{Выводы}

На основе CFD прогнозирования с использованием программного обеспечения FLUENT выявлены закономерности изменения теплогидравлических характеристик сверхкритической воды в вертикальных гладких трубах с нагреваемой длиной 4 м и внутренним диаметром 10 мм при варьировании ее массовой скорости $G$ в

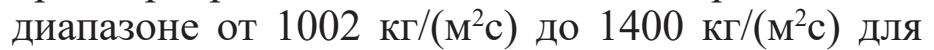
$P=23,9$ МПа и $q=681 \mathrm{\kappa BT} / \mathrm{M}^{2}$. Показано, что влияние массовой скорости на картину течения в трубе является неоднозначным ввиду действия ряда конкурирующих факторов. Ее увеличение приводит на значительном участке трубы, прилежащем к ее входу $(0<z<2,6$ м), к повышению скорости сверхкритической воды, а на участке, примыкающем к выходу трубы $(2,6 \leq z<4,0$ м) - к снижению скорости. Установлено также, что с ростом $G$ имеет место существенное увеличение коэффициента теплоотдачи $\alpha$ (в центральной части трубы до 2,25 раза) при реализации его значительно более неравномерного распределения по длине трубы. Показано, что движение фронта псевдокритического перехода от стенки трубы к ее оси при увеличении массовой скорости сверхкритической воды замедляется.

\section{ЛИТЕРАТУРА}

1. Кириллов П.Л. Водоохлаждаемые реакторы на воде сверхкритических параметров // Теплоэнергетика. - 2009, № 5. С. 2-5.

2. Жук В.В., Барбашев С.В. Кравченко В.П.
Обеспечение безопасности АЭС с реактором ВВЭР-СКД // Ядерна енергетика та довкілля. 2015, №1. - C.4 - 10.

3. Kelly J.E. Generation IV International Forum: A decade of progress through international cooperation. - Progress in Nuclear Energy. - 2014, Vol. 77. - P. $240-246$.

4. Глебов А.П., Терентьева М.И. Разработка прототипа реактора SCWR, охлаждаемого водой при сверхкритическом давлении, в рамках программы GIF (МФП-4) // Атомная техника за рубежом. - 2014, № 5. - С. 3-17.

5. Асмолов В.Г., Семченков Ю.М., Сидоренко В.А. Облик АЭС с легководными энергетическими реакторами следующего поколения //Седьмая международная научно-техническая конференция «Безопасность, эффективность и экономика атомной энергетики». - Москва, 26-27 мая 2010. M: ОАО "Концерн Росэнергоатом", 2010 C. 7-14.

6. Семченков Ю.М., Сидоренко В.А. Перспективы развития АЭС с ВВЭР // Теплоэнергетика. 2011, № 5. - C. 2-9.

7. Agranat V., Malin M., Pioro I., Abdullah R., Perminov V.A. CFD Modelling of Supercritical Water Heat Transfer in a Vertical Bare Tube Upward Flow // Proceedings of ICONE-23, May 17-21, Chiba, Japan. - 2015. - Paper 1163. - 11 p.

8. Vanyukova, G.V., Kuznetsov, Yu.N., Loninov A.Ya., Papandin M.V., Smirnov V.P. and Pioro I.L. Application of CFD Code to Calculations of Heat Transfer in a Fuel Bundle of SCW Pressure Channel Reactor . - Proc. 4th International Symposium on Supercritical Water-Cooled Reactors (ISSCWR-4), March 8-11, 2009. - Heidelberg, Germany, 2009. Paper 28. $-9 \mathrm{p}$.

9. Farah A., Harve, G. and Pioro I. Assessment of Fluent CFD Code as an Analysis Tool for Supercritical-Water Heat-Transfer Applications Proceedings of the 15th International Topical Meeting on Nuclear Reactor Thermalhydraulics (NURETH-15), Pisa, Italy, May 12-15 2013. - Paper 118. $-13 \mathrm{p}$.

10. Фиалко Н.М., Пиоро И.Л., Майсон Н.В., Меранова Н.О. Моделирование течения и теплообмена в гладких трубах при сверхкритических давлениях// Промышленная теплотехника. - 2016, №3. - C.12-21. 
11. Mokry S., Pioro I.L., Farah A., King K., Gupta S., Peiman, W. and Kirillov P. Development of Supercritical Water Heat-Transfer Correlation for Vertical Bare Tubes// Nuclear Engineering and Design. - 2011, Vol. 241. - P. 1126-1136.

\section{EFFECT OF MASS FLUX ON FLOW CHARACTERISTIC AND HEAT TRANSFER IN BARE TUBES AT SUPERCRITICAL PARAMETERS}

Fialko N.M. ${ }^{1,3}$, Pioro I.L. ${ }^{2}$, Maison N.V. ${ }^{1}$, Meranova N.O. ${ }^{1}$, Sharaevsky I.G. ${ }^{3}$

${ }^{1}$ Institute of Engineering Thermophysics of the National Academy of Sciences of Ukraine, Zhelyabova 2a, Kyiv, 03057, Ukraine

${ }^{2}$ Faculty of Energy Systems and Nuclear Science University of Ontario Institute of Technology 2000 Simcoe Str. N., Oshawa ON L1K 7K4 Canada

${ }^{3}$ Institute of safety problem of NPP of the National Academy of Sciences of Ukraine, Lysogorskaja, 12, Kyiv, 03680, Ukraine

On the basis of CFD of prediction with the use of FLUENT software the regularities of changes of supercritical water thermal and hydraulic characteristics by varying its mass flux in the range from $1002 \mathrm{~kg} / \mathrm{m}^{2} \mathrm{~s}$ up to $1400 \mathrm{~kg} / \mathrm{m}^{2} \mathrm{~s}$ for inlet pressure $P=23,9 \mathrm{MPa}$ and wall heat flux $q=681 \mathrm{~kW} / \mathrm{m}^{2}$ were revealed. It is shown that the effect of the mass flux on the flow pattern in the tube is ambiguous due to the action of a number of competing factors. The mass flux increase leads to the supercritical water velocity increasing on considerable section of the tube adjacent to its inlet $(0<z<2,6 \mathrm{~m})$, and on the section adjacent to the outlet of the tube $(2,6 \leq z<4,0 \mathrm{~m})-$ to the velocity decreasing. The fact that with increasing $G$ significant increasing of $\alpha$ (at the central part of the tube to 2,25 times) has place at realization of its much more irregular distribution along the tube length was also found. It was shown that the pseudocritical transition front movement from the tube wall to its axis slows at increasing the mass flux.

References 11, figures 6. 
Key words: Supercritical Water, Computational Fluid Dynamics (CFD), Flow and Heat Transfer, Mass flux.

1. Kirillov P.L. Water-cooled reactors on supercritical water. Teploenergetika. - 2009, № 5 . P. 2-5. (Rus)

2. Zhuk V.V., Barbashev S.V., Kravchenko V.P. Safety Assurance of NPP with VVER-SKD. Yaderna energetyka i dovkillya. - 2015, №1. P.4-10. (Rus)

3. Kelly J.E. Generation IV International Forum: A decade of progress through international cooperation . - Progress in Nuclear Energy. - 2014, Vol. 77. - P. 240-246. (Eng)

4. Glebov A.P., Terentiev M.I. Development SCWR reactor prototype which is water-cooled at supercritical pressure under the GIF program (IFP-4). - Atomnaia technika za rubezhom. - 2014, № 5. -P. 3-17. (Rus)

5. Asmolov V.G., Semchenkov Yu.M., Sidorenko $V$.A. Look NPP with light-water power reactors of the next generation. - Sedmaya mezhdunarodnaya nauchno-tehnicheskaya konferentsiya «Bezopasnost, effektivnost i ekonomika atomnoy energetiki». Moskva, 26-27 maya 2010. - M: OAO «Kontsern Rosenergoatom», 2010. - P. 7-14. (Rus)

6. Semchenkov Yu. M., Sidorenko V.A. Prospects for the development of nuclear power plants with VVER . - Tepoenergetika. - 2011, № 5. - P. 2-9. (Rus)
7. Agranat V., Malin M., Pioro I., Abdullah R., Perminov V.A. CFD Modelling of Supercritical Water Heat Transfer in a Vertical Bare Tube Upward Flow. Proceedings of ICONE-23, May 17-21, Chiba, Japan. - 2015. - Paper 1163. - 11 p. (Eng)

8. Vanyukova G.V., Kuznetsov Yu.N., Loninov A.Ya., Papandin M.V., Smirnov V.P. and Pioro I.L. Application of CFD Code to Calculations of Heat Transfer in a Fuel Bundle of SCW Pressure Channel Reactor// Proc. 4th International Symposium on Supercritical Water-Cooled Reactors (ISSCWR-4), March 8-11, 2009. - Heidelberg, Germany, 2009. Paper 28. - 9 p. (Eng)

9. Farah A., Harvel G. and Pioro I. Assessment of Fluent CFD Code as an Analysis Tool for Supercritical-Water Heat-Transfer Applications. Proceedings of the 15th International Topical Meeting on Nuclear Reactor Thermalhydraulics (NURETH-15), Pisa, Italy, May 12-15 2013. - Paper 118. - 13 p. (Eng)

10. Fialko N.M., Pioro I.L., Mayson N.V., Meranova N.O. Simulation of flow and heat transfer in the bure tubes at supercritical pressures. Promyshlennaia teplotehnika. - 2016. - №3. P. 12-21. (Rus)

11. Mokry S., Pioro I.L., Farah A., King K., Gupta S., Peiman W. and Kirillov P. Development of Supercritical Water Heat-Transfer Correlation for Vertical Bare Tubes. - Nuclear Engineering and Design. - 2011, Vol. 241. - P. 1126-1136. (Eng)

Получено 16.06.2016 Received 16.06.2016 